\title{
Targeting tumor microenvironment as a treatment strategy for hepatocellular carcinoma
}

\author{
Zuyuan Lin ${ }^{1,2 \#}$, Xiaonan Xiang ${ }^{1,2 \#}$, Di Lu ${ }^{1,2}$, Xiao Xu ${ }^{1,2}$ \\ ${ }^{1}$ Department of Hepatobiliary and Pancreatic Surgery, First Affiliated Hospital, Zhejiang University School of Medicine, Hangzhou, China; \\ ${ }^{2}$ National Clinical Research Center for Infectious Diseases, Hangzhou, China \\ \#These authors contributed equally to this work. \\ Correspondence to: Xiao Xu. Department of Hepatobiliary and Pancreatic Surgery, First Affiliated Hospital, Zhejiang University School of Medicine, \\ 79 Qingchun Road, Hangzhou 310003, China. Email: zjxu@zju.edu.cn.
}

Submitted Feb 17, 2020. Accepted for publication Mar 13, 2020.

doi: 10.21037/hbsn.2020.03.24

View this article at: http://dx.doi.org/10.21037/hbsn.2020.03.24

Liver cancer was ranked the sixth most commonly diagnosed cancer with the fourth leading cause of cancer-related deaths worldwide in 2018 (1). Notably, hepatocellular carcinoma (HCC) constitutes $75-85 \%$ among all the liver cancer cases, and ranks first morbidity and mortality of malignancies in Chinese males under 60. Due to hypervascularity in liver and high heterogeneity, HCC was regarded as an aggressive disease. Recent clinical trials focusing on resection and transplantation after targeted therapy for advanced HCC show encouraging results. However, the adjuvant therapy for patients with high risk of recurrence after surgery still raises attention. Currently, emerging evidence suggested that the crosstalk between tumor cells and the microenvironment plays a pivotal role in tumorigenesis and metastasis.

The tumor microenvironment of HCC provides tumor cells with necessary conditions for their sustained growth, invasion and metastasis. It is typically characterized by dysregulated immune network, sustained injury and regeneration, angiogenesis, inflammation and metabolic reprogramming. These aforementioned characteristics raise attention to the important role of infiltrating immune cell profile and tumor stroma in microenvironmental regulation. According to the immune cell subset and proportion, HCC could be classified into different immune subtypes, for example, Sia's "immune class/ exclusion" and Kurebayashi's "immune high/mid/low". Zhang et al. further identified three novel distinctive HCC subtypes with immunocompetent, immunodeficient, and immunosuppressive features (2). Immunocompetent and immunosuppressive subtype were characterized by robust immune cell infiltration and upregulated immune checkpoint molecules. These two subtypes, as well as the "immune class" and "immune-high subtype", displayed significant increase in immune cells infiltration and better responsiveness to immunotherapy. Tumor stroma and vasculature could also cause considerable impact on tumor progression. Cancer-associated fibroblasts (CAFs) can result in a robust stromal reaction characterized by fibrotic extracellular matrix and make tumor cells convert to aggressive and immune-excluded phenotype via transforming growth factor- $\beta$ (TGF- $\beta$ ) signaling pathway (3). Tumor-associated vasculature is another main hallmark of cancers. Endothelial cells (ECs) of the tumor vasculature can not only inhibit anti-tumor immunity by immune cells regulation and elimination, but also help to create a proangiogenic microenvironment via vascular endothelial growth factor (VEGF) signaling pathway (4). The disorganized tumor vasculature could remodel a special microenvironment characterized by hypoxia, acidosis, and high interstitial pressure, which both support tumor cells proliferation and structure a physical barrier for infiltrating immune cells (5). Furthermore, the interaction between different components relies on the regulation of chemokines and cytokines, which play essential roles in recruiting immune cells and maintaining cancer and stromal cell biology (6). In another word, these microenvironmental populations profoundly influence the tumor immune and metabolic homeostasis, which suggests immunometabolism as a novel perspective of HCC microenvironment. 
The concept of immunometabolism has highlighted the significance of crosstalk between immune microenvironment and metabolic reprogramming rather than isolated immune or metabolic alteration. For instance, macrophage plays a leading role in the immunometabolic microenvironment of HCC due to its quantity. On the one hand, it could be activated by metabolic signals and subsequently stimulated hepatocyte lipogenesis and cell death. On the other, the metabolic alteration in macrophages could further lead to immune reaction suppression, tumor proliferation and angiogenesis (7). Hence, interruption of those immunometabolism-associated cells or molecules would be a new direction of targeting tumor microenvironment for the treatment of HCC.

The possibility of targeting immunometabolism for tumor therapy is extensively studied in T cells (8). Selective alteration of metabolic process such as glycolysis or oxidative phosphorylation could help rescue $\mathrm{T}$ cell exhaustion within microenvironment and in turn improve the efficacy of checkpoint inhibitors (9). As for adoptive cell transfer, $T$ cells could be metabolically manipulated in vitro to promote mitochondrial integrity and oxidative metabolism, which would better support their in vivo anti-tumor function and longevity (9). Metabolic reprogramming of innate immune cells also provides options to restore normal regional immune surveillance. Recent work has shown that glycolytic enzyme PFKFB3 was a key driver of PD-L1 in macrophages, and thus conducted $\mathrm{T}$ cell exhaustion in HCC, which could be reversed by inhibition of PFKFB3 (10). And intracellular accumulation of cholesterol was known to have resulted in activated effector function of NK cells against hepatoma cells. Strategies to increase cholesterol uptake by NK cells might therefore be developed for treatment of HCC (11). These findings suggest that key metabolic molecules including enzymes and metabolites have the potential to be "immunometabolic checkpoints" that reprogram immune cells metabolically into an antitumor phenotype and support traditional immunotherapies like PD-1/L1 inhibitor or CAR-T.

However, targeting the primary tumor and its surrounding microenvironment is just the "first round" of tumor treatment. There is yet a distance to tumor eradication, due to the inevitable invasion and metastasis. Hence, we carefully proposed the attention to preventing secondary tumor microenvironment formation as well, that is, pre-metastatic niche. The pre-metastatic niche can be defined as a supportive "soil" which is undergoing the microenvironment profile changes to form the specific sites in preparation for metastasis, which can also be regarded as the extension of primary microenvironment. Targeting the pre-metastatic niche-promoting components to suppress pre-metastatic niche formation can consequently result in the "imprisonment" of HCC. Disabling its invasiveness provides valuable treatment time window for patients who are waiting for transplantation or those who are unable to sustain surgical damage. In these settings, targeting the molecular events evolved in multiple steps of metastasis provides options for us to interfere these processes. Several signaling axes including stromal-derived factor-1 (SDF1)/C-X-C motif chemokine receptor 4 (CXCR4) and interleukin-6 (IL-6)/signal transducer and activator of transcription 3 (STAT3) have been reported to be crucial for pre-metastatic niche formation in pre-clinical animal models $(12,13)$. Blocking the delivery of metastasispromoting exosomes to recipient cells may be another effective strategy for preventing HCC metastasis due to their roles in educating the pre-metastatic niche (14). Given the complicated composition within exosomes, deeper understanding of exosomes' role in metastasis and a more specific targeting tool are needed.

Besides pre-metastatic niche, some researchers have also bestowed their attention to field cancerization, which is defined as the cancer-primed cell population that underlies the development of many cancers, albeit presented without morphological changes (15). The surrounding microenvironment may be altered by the cancerized field and cancerization would progress in an appropriate microenvironmental context. Nowadays, the clinical issue presented by field cancerization mainly focused on risk prediction and surgical margin decision. Understanding the evolution of cells and surrounding microenvironment within these cancerized fields may make it possible to intervene ahead of tumor progression.

To date, the fundamental researches have already revealed the important role of tumor microenvironment in tumorigenesis and metastasis. A better understanding of microenvironmental regulation will facilitate a breakthrough in precision medicine and provide a rationale for clinical study. Besides that, targeting pre-metastatic niche as an adjuvant therapy will besiege tumor cells locally by disabling their invasions. This approach hopefully provides time window for patients to receive curative surgical treatment. It is promising that further investigation on the mechanism of pre-metastatic niche formation, even the cancerized field would provide a new insight into the 
prevention and treatment of HCC.

\section{Acknowledgments}

We thank Dr. Jianguo Wang for his advices on the manuscript and Dr. Winyen Chen for the text revision.

Funding: This work was supported by fundings from National Natural Science Foundation of China (No. 81930016), National Major Scientific and Technological Special Project (2017ZX10203205) and Key Research \& Development Plan of Zhejiang Province (2019C3050).

\section{Footnote}

Provenance and Peer Review: This article was commissioned by the editorial office of Hepatobiliary Surgery and Nutrition. The article did not undergo external peer review.

Conflicts of Interest: All authors have completed the ICMJE uniform disclosure form (available at http://dx.doi. org/10.21037/hbsn.2020.03.24). The authors have no conflicts of interest to declare.

Ethical Statement: The authors are accountable for all aspects of the work in ensuring that questions related to the accuracy or integrity of any part of the work are appropriately investigated and resolved.

Open Access Statement: This is an Open Access article distributed in accordance with the Creative Commons Attribution-NonCommercial-NoDerivs 4.0 International License (CC BY-NC-ND 4.0), which permits the noncommercial replication and distribution of the article with the strict proviso that no changes or edits are made and the original work is properly cited (including links to both the formal publication through the relevant DOI and the license). See: https://creativecommons.org/licenses/by-nc-nd/4.0/.

\section{References}

1. Bray F, Ferlay J, Soerjomataram I, et al. Global cancer statistics 2018: GLOBOCAN estimates of incidence and mortality worldwide for 36 cancers in 185 countries. CA Cancer J Clin 2018;68:394-424.

2. Zhang Q, Lou Y, Yang J, et al. Integrated multiomic analysis reveals comprehensive tumour heterogeneity and novel immunophenotypic classification in hepatocellular carcinomas. Gut 2019;68:2019-31.
3. Galluzzi L, Chan TA, Kroemer G, et al. The hallmarks of successful anticancer immunotherapy. Sci Transl Med 2018. doi: 10.1126/scitranslmed.aat7807.

4. Saharinen P, Eklund L, Pulkki K, et al. VEGF and angiopoietin signaling in tumor angiogenesis and metastasis. Trends Mol Med 2011;17:347-62.

5. Schaaf MB, Garg AD, Agostinis P. Defining the role of the tumor vasculature in antitumor immunity and immunotherapy. Cell Death Dis 2018;9:115.

6. Nagarsheth N, Wicha MS, Zou W. Chemokines in the cancer microenvironment and their relevance in cancer immunotherapy. Nat Rev Immunol 2017;17:559-72.

7. Krenkel O, Tacke F. Liver macrophages in tissue homeostasis and disease. Nat Rev Immunol 2017;17:306-21.

8. Geeraerts X, Bolli E, Fendt SM, et al. Macrophage Metabolism As Therapeutic Target for Cancer, Atherosclerosis, and Obesity. Front Immunol 2017;8:289.

9. Chang CH, Pearce EL. Emerging concepts of T cell metabolism as a target of immunotherapy. Nat Immunol 2016;17:364-8.

10. Chen DP, Ning WR, Jiang ZZ, et al. Glycolytic activation of peritumoral monocytes fosters immune privilege via the PFKFB3-PD-L1 axis in human hepatocellular carcinoma. J Hepatol 2019;71:333-43.

11. Qin WH, Yang ZS, Li M, et al. High Serum Levels of Cholesterol Increase Antitumor Functions of Nature Killer Cells and Reduce Growth of Liver Tumors in Mice. Gastroenterology 2020;158:1713-27.

12. Yang P, Hu Y, Zhou Q. The CXCL12-CXCR4 signaling axis plays a key role in cancer metastasis and is a potential target for developing novel therapeutics against metastatic cancer. Curr Med Chem 2020;27:5543-61.

13. Jing B, Wang T, Sun B, et al. Interleukin-6/STAT3 Signaling Orchestrates Pre-metastatic Niche Formation and Immunosuppressive Traits in Lung. Cancer Res 2020;80:784-97.

14. Guo Y, Ji X, Liu J, et al. Effects of exosomes on premetastatic niche formation in tumors. Mol Cancer 2019;18:39.

15. Curtius K, Wright NA, Graham TA. An evolutionary perspective on field cancerization. Nat Rev Cancer 2018;18:19-32.

Cite this article as: Lin Z, Xiang X, Lu D, Xu X. Targeting tumor microenvironment as a treatment strategy for hepatocellular carcinoma. Hepatobiliary Surg Nutr 2020;9(6):794796. doi: $10.21037 / \mathrm{hbsn} .2020 .03 .24$ 